Article

\title{
Synthesis of High Molecular Weight Polyester Using in Situ Drying Method and Assessment of Water Vapor and Oxygen Barrier Properties
}

\author{
Shouyun Cheng $\mathbb{D}^{\mathbb{D}}$, Burhan Khan $\mathbb{D}^{\mathbb{D}}$, Fahad Khan and Muhammad Rabnawaz *(D) \\ School of Packaging, Michigan State University, East Lansing, MI 48824-1223, USA; \\ shouyun.cheng@sdstate.edu (S.C.); khanburh@msu.edu (B.K.); fahad438@gmail.com (F.K.) \\ * Correspondence: rabnawaz@anr.msu.edu; Tel.: +1-517-432-4870
}

Received: 21 September 2018; Accepted: 7 October 2018; Published: 9 October 2018

\begin{abstract}
The preparation of renewable polyesters with good barrier properties is highly desirable for the packaging industry. Herein we report the synthesis of high molecular weight polyesters via an innovative use of an in situ drying agent approach and the barrier properties of the films formed from these polyesters. High number average molecular weight $\left(M_{n}\right)$ semiaromatic polyesters (PEs) were synthesized via alternating ring-opening copolymerization (ROCOP) of phthalic anhydride (PA) and cyclohexene oxide (CHO) using a salen chromium(III) complex in the presence of 4-(dimethylamino)pyridine (DMAP) cocatalyst. The use of a calcium hydride (drying agent) was found to enhance the number $M_{n}$ of the synthesized PEs, which reached up to $31.2 \mathrm{ku}$. To test the barrier properties, PE films were prepared by solvent casting approach and their barrier properties were tested in comparison poly(lactic acid) films. The PE films showed significantly improved water vapor and oxygen barrier properties compared to the commercial poly(lactic acid) (PLA) film that suggests the potential use of these PEs in in the food packaging industry.
\end{abstract}

Keywords: polyesters; salen chromium(III) complex; alternating ring-opening copolymerization; food packaging

\section{Introduction}

Polyesters (PEs), especially those derived from renewable resources, have attracted great interest because they are considered as an alternative to the current petrochemical-based plastics [1]. Polyesters are widely used in environmental, pharmaceutical, and commodity materials, and they are employed in packaging applications, due to their excellent barrier properties [2]. Polyesters can be produced via polycondensation or ring-opening copolymerization (ROCOP) methods [1]. The polycondensation method for polyester synthesis involves the reaction of diols with diacids (or diesters) [3]. However, this method is energy-intensive, since it requires high temperatures to induce condensation, reduced pressure to remove water or methanol and longer reaction periods to achieve high molecular weight polyesters [3].

The ring-opening copolymerization method involves a polymerization reaction between epoxides and cyclic anhydrides [4]. A benefit of ring-opening polymerizations is that they can be performed at mild temperatures without any need for the vacuum removal of the methanol/water molecules prepared during a condensation polymerization. Therefore, this reaction provides a greener synthetic approach for polyester synthesis compared to the polycondensation method. Many anhydrides and epoxides, including phthalic anhydride (PA), succinic anhydride (SA), cyclohexanedicarboxylic anhydride (CHA), maleic anhydride (MA), cyclohexene oxide (CHO), and epichlorohydrin $(\mathrm{ECH})$ have been derived from renewable resources and are employed as feedstock for the ring-opening 
polymerization method to produce green Pes [5]. However, the ROCOP approach is not yet mature, and suffers from limitations, such as low $M_{n}$ polymers and low turn-over frequency [2].

In order to increase the molecular weight of PE synthesized via the ring-opening polymerization method, many different metal-salen ligand catalysts and cocatalysts have been developed for the ROCOP of epoxides and anhydrides, including zinc [6], magnesium [7], chromium [8], cobalt [9], manganese [10], iron [11], aluminum [12], and nickel complex [13], most of which showed significantly higher activity in the presence of a nucleophilic cocatalyst, such as 4-dimethylaminopyridine (DMAP), bis(triphenylphosphine)iminium salts ([PPN]X), phosphines and ammonium salts. Duchateau and coworkers conducted a comprehensive study on the copolymerization of cyclohexene oxide (CHO) with phthalic anhydride (PA), succinic anhydride (SA), and cyclopropane-1,2-dicarboxylic acid anhydride (CAA) using salen aluminum, chromium and cobalt complexes in combination with several cocatalysts, such as bis(triphenylphosphine) iminium chloride (PPNCl) and 4-(dimethylamino)pyridine (DMAP) [14]. The highest catalytic activity was obtained by the combination of a salen chromium(III) complex catalyst with DMAP cocatalyst. The most reactive substrate was PA, which produced PEs with the highest molar mass (13 ku). Coates et al. [15] also found that salen chromium(III) complex was an effective catalyst for the ring-opening copolymerization of propylene oxide and maleic anhydride to produce high $M_{n}$ poly(propylene fumarate) $\left(17 \mathrm{ku}\right.$ ). However, the $M_{\mathrm{n}}$ values of aromatic polyesters are still not sufficiently and that is the possible reasons to for not testing the barrier properties of these PE films.

A key reason for the low $M_{n}$ of PE produced by ROCOP method is the presence of residual water impurity that functions as the chain transfer agent [16]. The residual water molecules originates from the reactants (i.e., epoxides and cyclic anhydrides) [14], even if they have been dried and distilled in the presence of calcium hydride. We reasoned that the incorporation of a drying agent during the polymerization could help to remove the residual water from reactants mixture, which will help to suppress undesired chain transfer reactions during the ROCOP. To the best of our knowledge, there have been no reports on the use of in situ drying agent (e.g., $\left.\mathrm{CaH}_{2}\right)$ to improve the molecular weights of aromatic/aliphatic PEs synthesized via ROCOP.

Poly(lactic acid) (PLA) is an aliphatic PE that is prepared from lactide by ring opening polymerization. PLA use in the packaging is increasing, since it is biobased, as well as compostable $[17,18]$. However, PLA has only moderate oxygen and water vapor permeation properties [1]. The development of bio-based PEs with improved oxygen and water vapor barrier properties for food packaging is highly desirable, since the enhanced barrier properties of packaging materials extend the shelf-lifetimes of perishable food items.

In this study, PE was synthesized via the ROCOP of $\mathrm{CHO}$ and PA, and then it was subsequently used to prepare films. The oxygen and water vapor permeation properties of these PE films were then compared with those of PLA films. We chose $\mathrm{CHO}$ and PA as precursors, because synthetic approaches exist to prepare $\mathrm{CHO}$ and PA from biobased triglycerides and carbohydrates, respectively $[19,20]$. In this research, an in situ drying agent was used for the first time during the ROCOP of CHO and PA. In the presence of calcium hydride drying agent, high $M_{n}$ PEs were synthesized. These PEs were characterized via NMR, GPC, FTIR and DSC techniques. The PE with the highest $M_{n}$ was employed to prepare PE films using a solvent casting method and their water vapor and oxygen barrier properties were investigated and compared to those of PLA films. To the best of our knowledge, the barrier properties of the PEs prepared by the ROCOP approach have never been reported.

\section{Experimental Section}

Materials. Cyclohexene oxide ( $\mathrm{CHO}$, Aldrich, St. Louis, MO, USA) was dried and distilled over calcium hydride $\left(\mathrm{CaH}_{2}\right.$, reagent grade $>95 \%$, Sigma Aldrich), and then degassed via three freeze-pump-thaw cycles and stored under nitrogen in a glovebox. Phthalic anhydride (PA, Aldrich) was recrystallized from dichloromethane (DCM, Aldrich) via a literature method [16]. 4-(Dimethylamino) pyridine (DMAP, Aldrich) was used as received. Toluene (Fisher), tetrahydrofuran 
(THF, Aldrich) and dichloromethane were collected from the solvent purification system. The salen chromium(III) complex((R,R)-N,N'-bis(3,5-di-tert-butylsalicylidene)-1,2-cyclohexanediaminochromium(III) chloride, $\mathrm{C}_{36} \mathrm{H}_{52} \mathrm{ClCrN}_{2} \mathrm{O}_{2}$, Aldrich) was used as received. Poly (96\% L-lactic acid) (PLA) with a $M_{n}$ of $112.54 \mathrm{ku}$ was purchased from NatureWorks LLC (Minnetonka, MN, USA) and used for film formation.

ROCOP reaction of $\mathrm{CHO}$ and PA. An $8 \mathrm{~mL}$ vial equipped with a magnetic stirring bar was charged with $\mathrm{CHO}$ ( 5 or $9 \mathrm{mmol})$, PA $(5 \mathrm{mmol})$, salen $\mathrm{Cr}$ catalyst $(10 \mu \mathrm{mol})$, DMAP cocatalyst $(10 \mu \mathrm{mol})$, toluene $(0.9 \mathrm{~mL})$ and $\mathrm{CaH}_{2}(0 \mathrm{mmol}$ or $2 \mathrm{mmol})$. The vial was then placed into a preheated aluminum heating block in the glove box. The copolymerization reaction was performed at $110{ }^{\circ} \mathrm{C}$. After the desired polymerization time of $22 \mathrm{~h}$, an aliquot of the crude reaction mixture was collected for ${ }^{1} \mathrm{H} N M R$ analysis to determine the conversion degree of $\mathrm{CHO}$. The rest of the reaction mixture was diluted with dry THF $(2 \mathrm{~mL})$, filtered through a $0.45 \mu \mathrm{m}$ PTFE syringe filter (Acrodisc) to remove $\mathrm{CaH}_{2}$, and precipitated from hexane $(20 \mathrm{~mL})$. The obtained PEs were centrifuged and dried in a rotary evaporator at room temperature $\left(20^{\circ} \mathrm{C}\right)$ for $2 \mathrm{~h}$ until a constant weight reached.

PE and PLA film preparation. PE and PLA were used to prepare films (Table 1 and Table S1 (Supplementary Materials)) for the barrier properties assessment using the following procedure. The $200 \mathrm{mg}$ of the selected polymer (PE and PLA) was dissolved in THF (0.4 mL). This solution was then cast onto an aluminum pan and covered by a glass jar to avoid the fast evaporation of THF and get films without defects and any perforations. Once the films were visibly dry (after $12 \mathrm{~h}$ at room temperature), the pans containing the PE or PLA films were shifted to an oven at $60{ }^{\circ} \mathrm{C}$ for $1 \mathrm{~h}$ to remove the residual THF. The pans were subsequently cooled to room temperature and the films were then detached from the pan, which were proceeded for evaluation of their water vapor and oxygen barrier properties.

Table 1. Films fabricated from polyester (PE), poly(lactic acid (PLA) or PLA-PE mixtures.

\begin{tabular}{cccc}
\hline Samples & PLA (mg) & PE (mg) & THF \\
\hline 1 & 200 & 0 & $0.4 \mathrm{~mL}$ \\
2 & 0 & 200 & $0.4 \mathrm{~mL}$
\end{tabular}

PE characterization. ${ }^{1} \mathrm{H}$ NMR spectra of the PE samples were recorded with a $500 \mathrm{MHz}$ NMR spectrometer (Agilent, Santa Clara, CA, USA) using chloroform- $d\left(\mathrm{CDCl}_{3}\right)$ as the solvent.

Molecular weights of the prepared PEs were determined using a Waters Breeze SEC (Size exclusion chromatography) RI (Refractive Index) chromatographic system (Waters 1515 pump, Waters 2410 refractive index detector and Waters 717plus Autosampler). GPC (Gel Permeation Chromatography) analysis were performed using four Styragel columns (HR1, HR2, HR3, and HR4) at $35^{\circ} \mathrm{C}$ in THF at an elution rate of $1 \mathrm{~mL} \cdot \mathrm{min}^{-1}$. Calibrations were performed using monodisperse polystyrene standards. As the GPC is a relative analytical tool to get the molecular weights, therefore all the polyesters were characterized under the same GPC conditions, using the same GPC method and GPC instrument. This enabled us to confidently compare the $M_{\mathrm{n}}$ and $Đ$ values for different polyesters.

Differential scanning calorimetry (DSC) analysis of the PEs was conducted in a Q100 differential scanning calorimeter (TA Instruments, New Castle, DE, USA) equipped with a mechanical cooling system.

The absorbance of the PEs was recorded in an Iraffinity-1S Fourier-transform infrared spectroscopy (FTIR, Shimadzu, Japan) instrument. The spectra were recorded over a range from 500 to $4000 \mathrm{~cm}^{-1}$. FTIR method mode is ATR. The polyester powder sample was put in the diamond tip and the pressed sample spectra was taken in ATR mode. The FTIR background signal was removed before sample FTIR analysis.

Water vapor and oxygen barrier properties. Water vapor and oxygen barrier performance of the film samples were measured using Permatran ${ }^{\circledR}$ model 3/33 and Ox-tran ${ }^{\circledR}$ model 2/21 permeation testing instruments (Mocon, Minneapolis, MN, USA), respectively. The film samples were mounted on 
aluminum foil masks with an exposed area of $0.18 \mathrm{~cm}^{2}$. Samples were tested at $50 \%$ relative humidity (RH) and $20^{\circ} \mathrm{C}$.

\section{Results and Discussion}

Scheme 1 Illustrates the PE synthesis via the ROCOP of CHO and PA in the presence of salen $\mathrm{Cr}$ (III) catalyst and DMAP cocatalyst. A detailed proposed mechanism is provided in the supporting information. In certain cases, $\mathrm{CaH}_{2}$ was added during polymerization as a drying agent. The synthesized PE was characterized by ${ }^{1} \mathrm{H}$ NMR (Figure 1), which showed four broad singlet peaks in the region of 1.13-2.16 ppm corresponding to four chemically different protons (axial and equatorial) of the cyclohexane ring. Similarly, the characteristics peak at $5.2 \mathrm{ppm}$ was assigned to $\mathrm{C}(\mathrm{O}) \mathrm{OCH}-$ protons, while the aromatic protons of the PA appeared between 7.4-7.6 ppm. In addition, the signal integrations of the two protons of $\mathrm{C}(\mathrm{O}) \mathrm{OCH}$ - and the four aromatic protons of the PA is essentially a 1:2 ratio, suggesting that $\sim 1: 1$ ratio of $\mathrm{CHO}$ and $\mathrm{PA}$ in the polyester. Similar assigning signals to specific protons of NMR spectra for polyester was reported in the study of Mundil et al. [11]. There are very weak signals in the ether linkage region (3.6 ppm) in the ${ }^{1} \mathrm{H}$ NMR spectrum (marked as "***") of $\mathrm{PE}$, which corresponds to the ether linkage formed by the two consecutive insertions of cyclohexene oxide during the polymerization. The very weak signals of the ether linkage in the ${ }^{1} \mathrm{H}$ NMR spectrum reveals that there is very little insertion of the consecutive cyclohexanone rings in the main alternating PE backbone. The catalytic mechanism of cyclohexene oxide (CHO) and phthalic anhydride (PA) copolymerization reaction using Salen $\mathrm{Cr}$ and -(dimethylamino)pyridine (DMAP) catalysts is shown in Scheme S1 (Supplementary Materials).
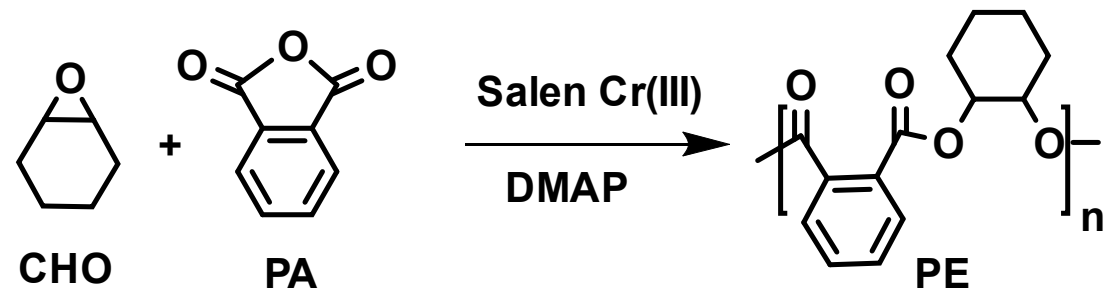

Scheme 1. Ring-opening copolymerization (ROCOP) reaction between cyclohexene oxide $(\mathrm{CHO})$ and PA in the presence of salen $\mathrm{Cr}(\mathrm{III})$ and -(dimethylamino)pyridine (DMAP).

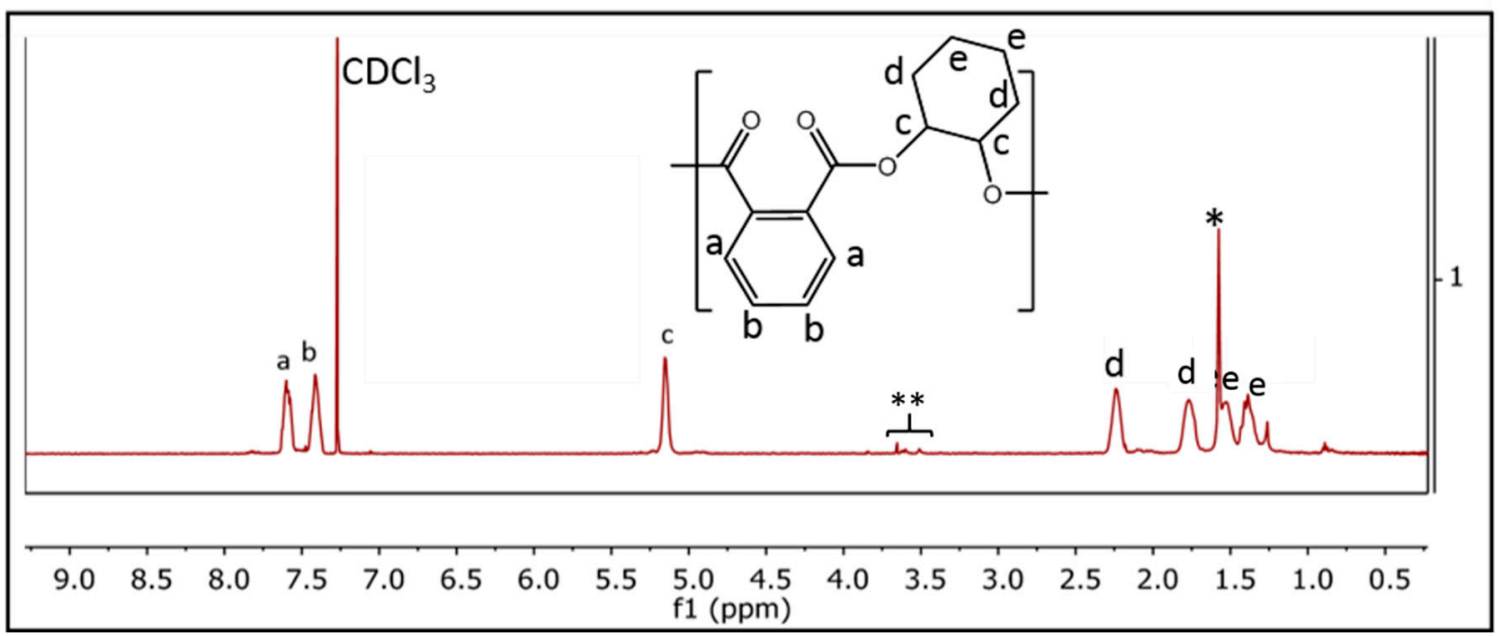

Figure 1. Representative ${ }^{1} \mathrm{H}$ NMR spectrum of the PE (entry\#3 in Table 2). * represents water impurity in the $\mathrm{CDCl}_{3}$ solvent, ${ }^{* *}$ represents ether linkage in the PE.

The preliminary tests of drying all the reagents were conducted to synthesize the polyester. However, the $M_{\mathrm{n}}$ of the polyester (5-6 ku) is much lower than the polyester synthesized in the presence 
of calcium hydride. Therefore, we conducted the test of synthesizing the polyester in the presence of both drying and calcium hydride. The ROCOP reaction between $\mathrm{CHO}$ and PA was investigated at different $\mathrm{CHO} / \mathrm{PA}$ ratios (1.0:1.0 and 1.8:1.0). The properties of the resultant PEs are summarized in Table 2. The CHO/PA ratio showed a significant influence on the $\mathrm{CHO}$ conversion ratio and the $M_{n}$ of the prepared PEs. A high $\mathrm{CHO}$ conversion ratio of $96.2 \%$ was obtained at the $\mathrm{CHO} / \mathrm{PA}$ ratio of 1.0:1.0 in the absence of $\mathrm{CaH}_{2}$. This $\mathrm{CHO}$ conversion ratio decreased to $67.9 \%$ with an increased $\mathrm{CHO} / \mathrm{PA}$ ratio (1.8:1.0) in the absence of $\mathrm{CaH}_{2}$. However, the $M_{n}$ of the PE increased significantly to $13.2 \mathrm{ku}$ at a $\mathrm{CHO} / \mathrm{PA}$ ratio of 1.8:1.0 compared to the value of $5.8 \mathrm{ku}$ that was obtained at a $\mathrm{CHO} / \mathrm{PA}$ ratio of 1.0:1.0. A similar trend has also been observed by Saini et al. [21].

The $M_{n}$ of the PE increased significantly in the presence of $\mathrm{CaH}_{2}$. For instance, in presence of $\mathrm{CaH}_{2}$, the $\mathrm{M}_{\mathrm{n}}$ of PE increased to 31.2 and $20.8 \mathrm{ku}$ at the $\mathrm{CHO} / \mathrm{PA}$ ratios of 1.0:1.0 and 1.8:1.0, respectively. This increase in the $\mathrm{M}_{n}$ possibly corresponds to the removal of water impurity by $\mathrm{CaH}_{2}$ and the suppression of subsequent chain transfer inhibitions. As shown in Table 2, all four of the synthesized PEs obtained exhibited a low polydispersity (1.06-1.10).

The thermal properties of these PEs were also investigated. It was found that the PEs exhibited enhanced thermal properties when their $\mathrm{M}_{\mathrm{n}}$ values increased. For example, the $T_{\mathrm{g}}$ of the PEs was elevated from 75 (entry 1) to $121^{\circ} \mathrm{C}$ (entry 2) as PE proceeded from the lowest to the highest $M_{n}$ of 5.8 and $31.2 \mathrm{ku}$, respectively. The $T_{\mathrm{g}}$ values of the PE reported in entry 3 and 4 were also very high $\left(\sim 110^{\circ} \mathrm{C}\right)$. These high $T_{\mathrm{g}}$ values for the Pes, shown in entries $2-4$ of Table 2 , suggest their potential for packaging use. The $T_{\mathrm{g}}$ of the PE in entry 3 was slightly increased relative to entry 4 PE despite entry $4 \mathrm{PE}$ has high $M_{\mathrm{n}}$. This anomaly is probably attributed to the high conversion of $\mathrm{CHO}(77.5 \%)$ in the PE of entry 4 compared to $67.92 \%$ in entry $3 \mathrm{PE}$. As CHO is flexible relative to PA, that's explain why the $T_{\mathrm{g}}$ was a bit lower for the entry 4 PE despite high $M_{\mathrm{n}}$.

Table 2. Properties of PEs synthesized from $\mathrm{CHO}$ and PA in the presence of salen $\mathrm{Cr}$ and DMAP a .

\begin{tabular}{cccccc}
\hline \multirow{2}{*}{ Entry } & $\mathbf{C H O} / \mathbf{P A} / \mathbf{C a H}_{\mathbf{2}}$ & $\mathbf{C H O}$ Conversion ${ }^{\mathbf{b}} \mathbf{( \% )}$ & \multicolumn{3}{c}{$\mathbf{P E}$} \\
\cline { 4 - 6 } & & & $\boldsymbol{M}_{\mathbf{n}}{ }^{\mathbf{c}} \mathbf{( k u )}$ & $\mathbf{\Xi}^{\mathbf{c}}$ & $\boldsymbol{T}_{\mathbf{g}}{ }^{\mathbf{d}}\left({ }^{\circ} \mathbf{C}\right)$ \\
\hline 1 & $1: 1: 0$ & 96.2 & 5.8 & 1.07 & 75 \\
2 & $1: 1: 0.4$ & 99.0 & 31.2 & 1.06 & 121 \\
3 & $1.8: 1: 0$ & 67.9 & 13.2 & 1.08 & 113 \\
4 & $1.8: 1: 0.4$ & 77.5 & 20.8 & 1.10 & 110 \\
\hline
\end{tabular}

a $\mathrm{T}=110{ }^{\circ} \mathrm{C} ; \mathrm{V}_{\text {toluene }}=0.9 \mathrm{~mL}$; salen Cr:DMAP:PA $=1: 1: 500 ;{ }^{\mathrm{b}}$ Determined by ${ }^{1} \mathrm{H}$ NMR spectroscopy; ${ }^{\mathrm{c}} M_{\mathrm{n}}$ (number average molecular weight) and $Đ$ (polydispersity) determined by SEC; ${ }^{d}$ Determined by differential scanning calorimetry (DSC).

The representative FTIR spectra of the PEs prepared in the presence and absence of $\mathrm{CaH}_{2}$ are shown in Figure S1 (Supplementary Materials). The FTIR spectrum of the PE confirms the ester linkages. For example, the band corresponding to the symmetric stretching vibration of the ester carbonyl $(\mathrm{C}=\mathrm{O})$ group appeared at $1720 \mathrm{~cm}^{-1}$ [22]. Meanwhile, the bands at 1250 and $1066 \mathrm{~cm}^{-1}$ corresponds to the asymmetric and symmetric stretching vibrations of the $\mathrm{C}-\mathrm{O}-\mathrm{C}$ group, respectively [11].

Barrier properties of the PE and Poly(lactic acid) (PLA) films. A key goal of this study is to demonstrate the barrier properties of the PEs prepared by ROCOP approach. The PE sample with a $M_{\mathrm{n}}$ of $31.2 \mathrm{ku}$ was used to prepare films for barrier performance tests. We chose this PE because of its high $M_{n}$. Representative images of the PE and PLA films used in this study are shown in Figure 2 and Figure S2 (Supplementary Materials). PLA film (Figure 2a) was used prepared as reference in this study.

The above films were subjected to water vapor and oxygen permeability tests. These tests were conducted because water vapor and oxygen barrier properties are the most significant factors that affect quality and shelf-life of a product [23]. The results obtain from the oxygen and water vapor permeabilities of the PE and PLA films are shown in Table 3 and Figures S3 and S4 (Supplementary Materials). The oxygen permeability of the PE film was $544 \mathrm{cc}-\mathrm{mil} /\left(\mathrm{m}^{2}\right.$-day-atm), which was 
considerably lower than that of the PLA film $1528 \mathrm{cc}-\mathrm{mil} /\left(\mathrm{m}^{2}\right.$-day-atm). This $62 \%$ reduction in oxygen permeability observed for the PE film compared to PLA could be attributed to relatively strong secondary interaction strengthened by the presence of aromatic benzene rings on PE chains as compared to PLA, which should theoretically have weak secondary interactions. The water vapor permeation of the PE film was $62 \mathrm{~g}$-mil/( $\mathrm{m}^{2}$-day-atm), which was significantly lower compared to that of the PLA film (168 g-mil/( $\mathrm{m}^{2}$-day-atm)). This $63 \%$ reduction in the water permeability of the PE films relative to PLA films is, again, probably due to the strong secondary interactions and closest chain packing of PE. Thus, the obtained PE films have shown a significant improvement in the water and oxygen barrier properties, which will provide these materials applications in the food packaging industry to protect perishable goods against oxygen and moisture degradation [24]. The water vapor and oxygen permeability of the PE $(0.18 \mathrm{~mm})$ was compared to another PLA film with similar thickness $(0.19 \mathrm{~mm})$. The water vapor and oxygen permeability the PE were still much lower than that of PLA film with similar thickness.
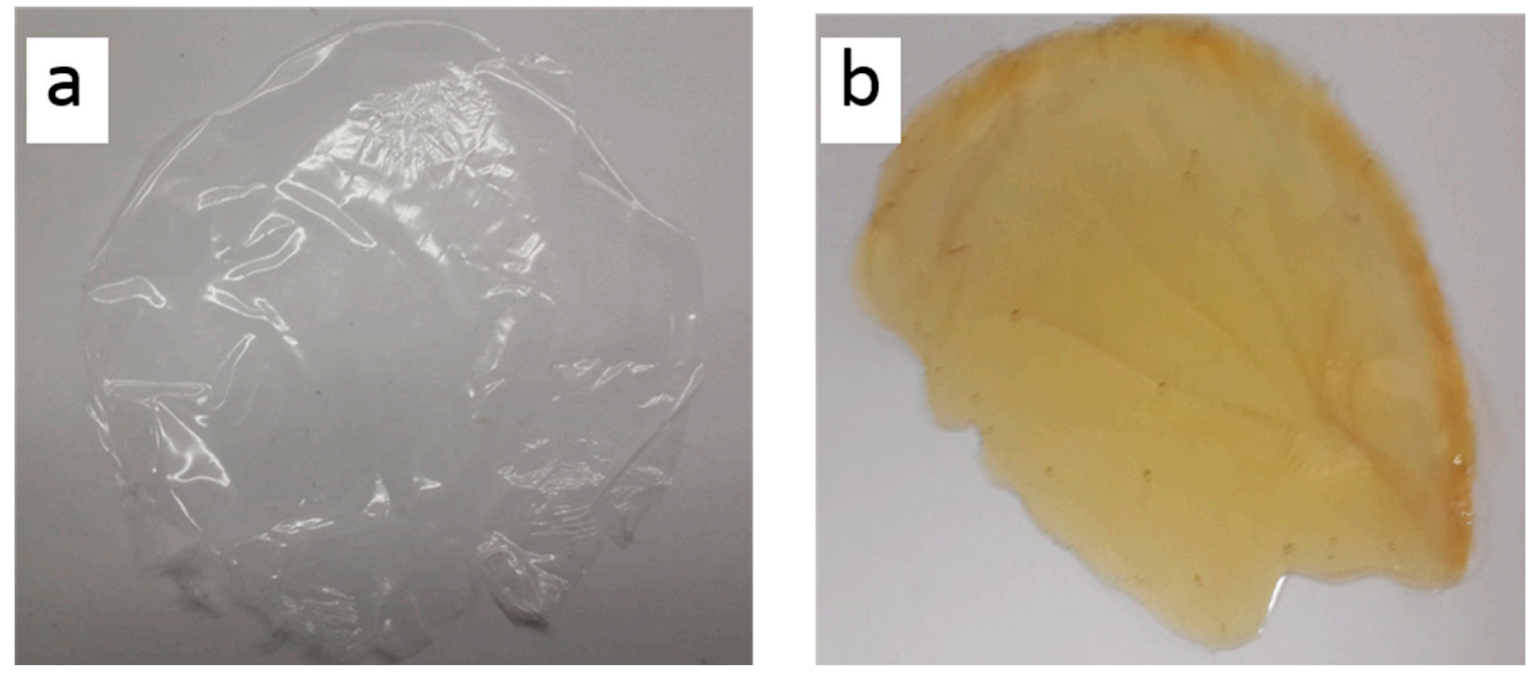

Figure 2. Images of PE and PLA films: (a) PLA; (b) PE.

Table 3. Permeability values for PLA and PE films.

\begin{tabular}{|c|c|c|c|}
\hline Sample & Thickness (mm) & $\begin{array}{l}\text { Oxygen Permeability } \\
\text { (cc. } \mathrm{mil} / \mathrm{m}^{2} \text {-day-atm) }\end{array}$ & $\begin{array}{l}\text { Water Vapor Permeability } \\
\text { (g. } \mathrm{mil} / \mathrm{m}^{2} \text {-day-atm) }\end{array}$ \\
\hline PLA & 0.04 & $1528 \pm 593.88$ & $168 \pm 62.03$ \\
\hline PLA & 0.19 & $1508 \pm 565.42$ & $148 \pm 12.10$ \\
\hline PE & 0.18 & $544 \pm 145.00$ & $62 \pm 5.01$ \\
\hline
\end{tabular}

The lower content of residual $\mathrm{CaH}_{2}$ in the obtained polymers is important. Therefore, after the polyester synthesis, the reaction mixture was diluted with dry THF $(2 \mathrm{~mL})$, filtered through a $0.45 \mu \mathrm{m}$ PTFE syringe filter to remove $\mathrm{CaH}_{2}$ and residual catalysts, and then precipitated from hexane $(20 \mathrm{~mL})$. The filtration and precipitation removed most of the $\mathrm{CaH}_{2}$ and residual catalysts. Besides, the residual $\mathrm{CaH}_{2}$ and catalysts could be further removed through filtration by silica or celite in the future studies. The obtained polyester was then cleaned through centrifuge and drying in a rotary evaporator at room temperature $\left(20^{\circ} \mathrm{C}\right)$ for $2 \mathrm{~h}$ to remove the residual solvents, such as THF and hexane. However, more repetitions of dissolving the polyester to THF, filtration through PTFE syringe filter and celite/silica, centrifuge and drying will be conducted to further clean polyester and remove the $\mathrm{CaH}_{2}$ or residual catalyst left in the final polyester.

The exposure to chromium compounds, such as ions $\mathrm{Cr}^{3+}$ and its organic ligands could result in the formation of ulcers, which will persist for months and heal very slowly [25]. The possible leaking chromium compounds in the food/cosmetic packaging might have this negative effect. 
However, the effect of chromium compound is limited, since only small ratio of chromium (salen Cr:DMAP:PA = 1:1:500) was used in polyester synthesis in this study. Most of the chromium catalyst was also removed during precipitation, washing, and filtration.

The thickness of the films used in a real application is around $2-3.2 \mathrm{~mm}$, which is similar to PLA film [26]. The 2-3.2 mm thickness of synthesized PEs could be made using casting method. Therefore, no obvious thresholds for the film thickness in case of the synthesized PEs was found yet. The PE film is stable in the drying temperature of $60^{\circ} \mathrm{C}$ for $1 \mathrm{~h}$, and it should be relatively stable, due to its relatively high $T_{\mathrm{g}}$ of $121^{\circ} \mathrm{C}$.

\section{Conclusions}

We demonstrated for the first time the in situ use of a drying agent to improve the $M_{n}$ of a PE. Aromatic polyesters (PEs) were synthesized via an in situ drying ROCOP reaction between $\mathrm{CHO}$ and PA using salen chromium(III) as a catalyst and 4-(dimethylamino)pyridine (DMAP) as a cocatalyst in the presence of $\mathrm{CaH}_{2}$. The use of a drying agent significantly increased the $M_{n}$ of the PEs. The NMR and FTIR spectra confirmed the chemical structure of PE. The highest $M_{n}$ of $31.2 \mathrm{ku}$ was synthesized at $\mathrm{CHO} / \mathrm{PA} / \mathrm{CaH}_{2}$ of 1:1:0.4. The $T_{\mathrm{g}}$ of this high $M_{\mathrm{n}} \mathrm{PE}$ was as high as $121^{\circ} \mathrm{C}$. We tested the barrier properties of a polymer prepared via the ROCOP approach for the first time. The barrier properties of the highest $M_{n}$ PE were evaluated against those of PLA. The PE films showed significant improvement in the barrier performance against oxygen and water vapor than was provided by PLA, which will encourage the possible use of these PE films for food packaging. The PE films also showed better oxygen and water vapor barrier performance than the PE and PLA mixture films. The negative effect of chromium compound is limited, due to the small ratio of chromium (salen Cr:DMAP:PA = 1:1:500) used in polyester synthesis.

Supplementary Materials: The following are available online at http:/ / www.mdpi.com/2073-4360/10/10/1113/ s1, Scheme S1: Catalytic mechanism cyclohexene oxide (CHO) and phthalic anhydride (PA) copolymerization reaction using Salen $\mathrm{Cr}$ and -(dimethylamino)pyridine (DMAP) catalysts, Figure S1: Representative 2. Fourier-transform infrared spectroscopy (FTIR) spectra of the polyester (PE) (entry 1 and 2 in Table 2), Table S1: Two films fabricated from poly(lactic acid) (PLA)-PE mixtures, Figure S2: Images of all the PE and PLA films (aPLA; b- PLA:PE (3:1); c- PLA:PE (1:1); d- PE), Figure S3: Oxygen barrier properties of all the PE and PLA films, Figure S4: Water vapor barrier properties of all the PE and PLA films.

Author Contributions: Conceptualization, S.C. and M.R.; Methodology, S.C. and F.K.; Writing-Original Draft Preparation, S.C. and M.R.; Revision, S.C., M.R. and B.K.

Conflicts of Interest: The authors declare no conflict of interest.

\section{References}

1. Rabnawaz, M.; Wyman, I.; Auras, R.; Cheng, S. A roadmap towards green packaging: The current status and future outlook for polyesters in the packaging industry. Green Chem. 2017, 19, 4737-4753. [CrossRef]

2. Liu, Y.; Guo, J.-Z.; Lu, H.-W.; Wang, H.-B.; Lu, X.-B. Making various degradable polymers from epoxides using a versatile dinuclear chromium catalyst. Macromolecules 2018, 51, 771-778. [CrossRef]

3. Vilela, C.; Sousa, A.F.; Fonseca, A.C.; Serra, A.C.; Coelho, J.F.; Freire, C.S.; Silvestre, A.J. The quest for sustainable polyesters-insights into the future. Polym. Chem. 2014, 5, 3119-3141. [CrossRef]

4. Longo, J.M.; Sanford, M.J.; Coates, G.W. Ring-opening copolymerization of epoxides and cyclic anhydrides with discrete metal complexes: Structure-property relationships. Chem. Rev. 2016, 116, 15167-15197. [CrossRef] [PubMed]

5. Ji, H.-Y.; Wang, B.; Pan, L.; Li, Y.-S. Lewis pairs for ring-opening alternating copolymerization of cyclic anhydrides and epoxides. Green Chem. 2018, 20, 641-648. [CrossRef]

6. Zhu, L.; Liu, D.; Wu, L.; Feng, W.; Zhang, X.; Wu, J.; Fan, D.; Lü, X.; Lu, R.; Shi, Q. A trinuclear $\left[\mathrm{Zn}_{3}(\mathrm{~L})_{2}(\mathrm{OAc})_{2}\right]$ complex based on the asymmetrical bis-Schiff-base ligand $\mathrm{H}_{2} \mathrm{~L}$ for ring-opening copolymerization of $\mathrm{CHO}$ and MA. Inorg. Chem. Commun. 2013, 37, 182-185. [CrossRef]

7. Winkler, M.; Romain, C.; Meier, M.A.; Williams, C.K. Renewable polycarbonates and polyesters from 1, 4-cyclohexadiene. Green Chem. 2015, 17, 300-306. [CrossRef] 
8. Darensbourg, D.J.; Poland, R.R.; Escobedo, C. Kinetic Studies of the Alternating Copolymerization of Cyclic Acid Anhydrides and Epoxides, and the Terpolymerization of Cyclic Acid Anhydrides, Epoxides, and $\mathrm{CO}_{2}$ Catalyzed by (salen)Cr ${ }^{\mathrm{III}} \mathrm{Cl}$. Macromolecules 2012, 45, 2242-2248. [CrossRef]

9. Nejad, E.H.; Paoniasari, A.; Koning, C.E.; Duchateau, R. Semi-aromatic polyesters by alternating ring-opening copolymerisation of styrene oxide and anhydrides. Polym. Chem. 2012, 3, 1308-1313. [CrossRef]

10. Liu, D.-F.; Zhu, L.-Q.; Wu, J.; Wu, L.-Y.; Lü, X.-Q. Ring-opening copolymerization of epoxides and anhydrides using manganese(III) asymmetrical Schiff base complexes as catalysts. RSC Adv. 2015, 5, 3854-3859. [CrossRef]

11. Mundil, R.; Hošt'álek, Z.; Šeděnková, I.; Merna, J. Alternating ring-opening copolymerization of cyclohexene oxide with phthalic anhydride catalyzed by iron (III) salen complexes. Macromol. Res. 2015, 23, 161-166. [CrossRef]

12. Bernard, A.; Chatterjee, C.; Chisholm, M.H. The influence of the metal (Al, $\mathrm{Cr}$ and $\mathrm{Co}$ ) and the substituents of the porphyrin in controlling the reactions involved in the copolymerization of propylene oxide and cyclic anhydrides by porphyrin metal(III) complexes. Polymer 2013, 54, 2639-2646. [CrossRef]

13. Yu, C.-Y.; Chuang, H.-J.; Ko, B.-T. Bimetallic bis(benzotriazole iminophenolate) cobalt, nickel and zinc complexes as versatile catalysts for coupling of carbon dioxide with epoxides and copolymerization of phthalic anhydride with cyclohexene oxide. Catal. Sci. Technol. 2016, 6, 1779-1791. [CrossRef]

14. Hosseini, N.E.; van Melis, C.G.; Vermeer, T.J.; Koning, C.E.; Duchateau, R. Alternating ring-opening polymerization of cyclohexene oxide and anhydrides: Effect of catalyst, cocatalyst, and anhydride structure. Macromolecules 2012, 45, 1770-1776. [CrossRef]

15. DiCiccio, A.M.; Coates, G.W. Ring-opening copolymerization of maleic anhydride with epoxides: A chain-growth approach to unsaturated polyesters. J. Am. Chem. Soc. 2011, 133, 10724-10727. [CrossRef] [PubMed]

16. Nejad, E.H.; Paoniasari, A.; van Melis, C.G.; Koning, C.E.; Duchateau, R. Catalytic ring-opening copolymerization of limonene oxide and phthalic anhydride: Toward partially renewable polyesters. Macromolecules 2013, 46, 631-637. [CrossRef]

17. Vink, E.T.; Rabago, K.R.; Glassner, D.A.; Gruber, P.R. Applications of life cycle assessment to NatureWorks ${ }^{\mathrm{TM}}$ polylactide (PLA) production. Polym. Degrad. Stab. 2003, 80, 403-419. [CrossRef]

18. Markarian, J. Biopolymers present new market opportunities for additives in packaging. Plast. Addit. Compd. 2008, 10, 22-25. [CrossRef]

19. Mahmoud, E.; Watson, D.A.; Lobo, R.F. Renewable production of phthalic anhydride from biomass-derived furan and maleic anhydride. Green Chem. 2014, 16, 167-175. [CrossRef]

20. Giarola, S.; Romain, C.; Williams, C.K.; Hallett, J.P.; Shah, N. Techno-economic assessment of the production of phthalic anhydride from corn stover. Chem. Eng. Res. Des. 2016, 107, 181-194. [CrossRef]

21. Saini, P.K.; Romain, C.; Zhu, Y.; Williams, C.K. Di-magnesium and zinc catalysts for the copolymerization of phthalic anhydride and cyclohexene oxide. Polym. Chem. 2014, 5, 6068-6075. [CrossRef]

22. Patil, S.G.; Bhadane, B.S.; Patil, M.P.; Belemkar, S.; Patil, R.H. In-vitro antioxidant activity, acute oral toxicity studies and preliminary phytochemical characterization of the bark extract of Terminalia arjuna (L.). J. Pharm. Nutr. Sci. 2016, 6, 15-21. [CrossRef]

23. Genovese, L.; Gigli, M.; Lotti, N.; Gazzano, M.; Siracusa, V.; Munari, A.; Dalla, R.M. Biodegradable long chain aliphatic polyesters containing ether-linkages: Synthesis, solid-state, and barrier properties. Ind. Eng. Chem. Res. 2014, 53, 10965-10973. [CrossRef]

24. Ren, P.-G.; Liu, X.-H.; Ren, F.; Zhong, G.-J.; Ji, X.; Xu, L. Biodegradable graphene oxide nanosheets/poly(butylene adipate-co-terephthalate) nanocomposite film with enhanced gas and water vapor barrier properties. Polym. Test. 2017, 58, 173-180. [CrossRef]

25. Jaishankar, M.; Tseten, T.; Anbalagan, N.; Mathew, B.; Beeregowda, K. Toxicity, mechanism and health effects of some heavy metals. Interdiscip. Toxicol. 2014, 7, 60-72. [CrossRef] [PubMed]

26. Oever, M.V.; Molenveld, K.; van der Zee, M.; Bos, H. Bio-Based and Biodegradable Plastics Facts and Figure; Wageningen Food \& Biobased Research: Wageningen, Holland, 2017.

(C) 2018 by the authors. Licensee MDPI, Basel, Switzerland. This article is an open access article distributed under the terms and conditions of the Creative Commons Attribution (CC BY) license (http:/ / creativecommons.org/licenses/by/4.0/). 